\title{
Las políticas de reorganización municipal y supranacional en Quebec
}

Raquel Ojeda García*

Palabras clave: Estructuras municipales y supramunicipales, descentralización, reagrupación municipal, Quebec.

El objeto de estudio de este artículo es la fusión de municipios en Quebec ciudad y la creación de la Comunidad Metropolitana de Quebec. Este estudio de caso se enmarca en un contexto más amplio, ya que la política de fusión de municipios y el refuerzo de las estructuras supramunicipales no afecta sólo a Quebec ciudad sino a toda la provincia ${ }^{1}$. El desarrollo de nuestro análisis está articulado en torno a los siguientes temas: en primer lugar, la presentación de las propuestas, escenarios y análisis para la reforma de la estructura municipal y supramunicipal; en segundo lugar, el estudio de los factores aducidos para la reorganización municipal, los costes y las posturas a favor y en contra de la fusión; en tercer lugar, la concreción e implantación de la política de reforma municipal y supramunicipal; en cuarto lugar, la construcción de la Comunidad Metropolitana de Quebec (definición, competencias, funciones, diseño y proyección); y por último, las políticas de reconstitución tras el cambio de Gobierno en Quebec provincia y sus consecuencias.

La hipótesis de trabajo es que la política de reforma municipal y supramunicipal está atravesada por tres ejes. El primero consiste en la pretensión de mejora de estos niveles de la Administración territorial desde una perspectiva de la gestión pública, con la finalidad sólo de mejorar el sistema fiscal y ganar en racionalidad y economías de escala. Esta política es promovida y llevada a cabo «desde arriba», impulsada por el Ministerio de Asuntos Municipales y de la Metrópolis. Sin embargo, nuestro objetivo es matizar esta idea con la introducción de la variable «política». En primer lugar, porque esta reforma puede ser analizada como una política pública y, en segundo lugar, porque todo Gobierno que ha intentado llevar a cabo una reforma en profundidad de la esfera local ha visto su poder dañado en las convocatorias electorales.

El segundo eje se centra en el marco de las reformas de reestructuración municipal, el proceso de descentralización. Las políticas de reagrupación municipal y supramunicipal están acompañadas de una política de descentralización ambigua desde el Gobierno de Quebec provincia. En las declaraciones formales del nivel central

\footnotetext{
* Profesora Contratada Doctora del Área de Ciencia Politica y de la Administración de la Universidad de Jaén.

${ }^{1}$ Quebec provincia es la denominación que se le da al Estado federado que forma parte de Canadá y es distinta a Quebec ciudad, la capital de la provincia.
} 
se pone de manifiesto la pretensión de profundizar en la descentralización política y administrativa. Sin embargo, esta pretensión se frena, por un lado, por la vinculación de la descentralización al logro de la Independencia en Quebec y, por otro, por la emergencia de una esfera local en donde se opta por las fusiones con unas instituciones elegidas de forma indirecta.

El tercer eje refleja que las políticas de reestructuración municipal se han enmarcado oficialmente dentro de una política global que refuerza la denominada legitimidad de la Administración por los rendimientos. Esta es la pretensión de la más reciente reforma de la Administración central y la publicación de la Ley sobre la Administración Pública en el año 2000 como marco para la gestión. La legitimidad institucional (basada en el ajuste de la actuación de la Administración a unos valores políticos socialmente aceptados) parece quedar relegada a un segundo plano. El factor lingüístico, en especial la defensa de la francofonía en el caso concreto de Montreal, cuestiona las declaraciones oficiales y las bases de la legitimidad fundadas sólo en los rendimientos de las actuaciones públicas.

Los objetivos de las políticas de reorganización municipal y supramunicipal son: la mejora de la funcionalidad del sistema, la simplificación de las estructuras y disminución de los cargos electos, el reequilibrio de la carga fiscal y el freno de la extensión urbana fuera de los cascos urbanos. Resulta muy difícil evaluar si estos objetivos se han cumplido, en primer lugar, por el poco tiempo transcurrido y, en segundo lugar, por los cambios introducidos por la política de reconstitución. Tras las elecciones de 2003 y el cambio de partido en el Gobierno se produce un giro radical en la política de la organización local y se aprueba la política de reconstitución. Esta decisión supone que los municipios reagrupados de forma obligatoria puedan reconstituirse como entes diferentes a la ciudad fusionada. Este cambio de la política de organización municipal y supramunicipal nos permite introducir la variable de la lengua como factor explicativo de las distintas reacciones. Este factor incide especialmente en el caso de la Comunidad Metropolitana de Montreal, ya que el porcentaje de hogares y personas que no utilizan como primera lengua el francés hace temer que Montreal deje de ser una gran ciudad de habla francesa en el continente americano. La tentación podría ser reducir la cuestión de las fusiones/reconstituciones a una cuestión lingüística, pero no podemos olvidar que la defensa de la francofonía supone en muchas ocasiones la reivindicación de una posición política defensora de la independencia de Quebec provincia.

Por último, abordamos la gestión metropolitana desde la óptica de la gobernabilidad, como una opción acorde con las exigencias actuales de las sociedades contemporáneas y como respuesta para la superación de un modelo de Administración weberiano incapaz de adaptarse a las reivindicaciones y demandas de los ciudadanos

El texto está basado en el análisis de los documentos oficiales publicados por el Ministerio de Asuntos Municipales y de la Metrópolis, la Comunidad Metropolitana de Quebec y la Ciudad de Quebec, así como en artículos publicados en la prensa (Le Devoir, Le Soleily La Presse), que reflejan las posturas tanto a favor como en contra de la fusión de los municipios, y en artículos de revistas científicas especializadas.

\section{Propuestas, escenarios y análisis para la reforma de la estruc- tura municipal y supramunicipal}

En el plano institucional, Canadá fue el primer país en experimentar la combinación de dos regímenes políticos, el federalismo y el sistema parlamentario de Westminster. Mientras que el federalismo implica dos niveles de gobierno teóricamente soberanos (el federal o estatal y el provincial) en sus campos de competencia, el parlamentarismo se caracteriza principalmente por una concentración del poder en la rama ejecutiva (Laforest y 
Montigny 2005: 348). De ahí que uno de los argumentos que se utilizara desde el Ministerio de Asuntos Municipales y de la Metrópolis de Quebec provincia para justificar su política de reorganización municipal fuera que los municipios canadienses, no importa a qué provincia pertenezcan, son «criaturas del gobierno», constitucionalmente desprovistos de autonomía e incluso de la garantía de su propia existencia desde 1867, fecha en la que se promulga la Constitution Act (Benoit y Mévellec 2003: 116). Para ciertos autores como Quesnel, esta afirmación deber ser matizada. Los gobiernos locales a pesar de depender de las asambleas provinciales gozan de una fuerte autonomía ya que el $90 \%$ de su financiación proviene de fuentes autónomas, forman parte de acuerdos institucionales de todo el sistema político y acaban por incidir en el Gobierno provincial (Quesnel 2000: 115).

La política de reforma municipal impulsada con mayor fuerza a partir de 1999 supone un desafío a la autonomía y a la identidad de los distintos municipios afectados. Para los defensores de esta política, los objetivos que justifican esta reforma consisten en una mejora de la gestión de las áreas metropolitanas para adaptarlas a los retos internacionales e internos, la consecución de una mayor equidad en la carga fiscal, una mejor y equilibrada prestación de servicios y reforzar las instituciones locales.

El diagnóstico de la situación de la organización municipal y supramunicipal en la década de los 90 comienza, en primer lugar, por la constatación de que la política municipal llevada a cabo ha sido bastante conservadora. Así lo revela el hecho de que el número de municipios no haya decrecido prácticamente en todo el siglo XX.

La estructura municipal de Quebec se construyó sobre la base de las parroquias y los pueblos desde la época colonial. Estas pequeñas poblaciones próximas al río San Lorenzo y otros ríos, han sido las que se han convertido en la actualidad en las ciudades más importantes de Quebec (Montreal, Quebec ciudad, Chicutimi, Hull, Trois-Rievières y Sherbrooke). Una de las características de la división municipal de Quebec provincia es su alta fragmentación, la más elevada de todo Canadá.

Si bien Montreal era la segunda área metropolitana de Canadá con 3,3 millones de habitantes, después de Toronto con 4,3 millones y por encima de Vancouver con 1,8 millones y Ottawa-Hull con 1 millón, también es cierto que el 90\% de los municipios de Quebec provincia antes de 2000 tenían menos de 10.000 habitantes y que sólo cinco ciudades superaban los 100.000 habitantes. En marzo de 2000, el número de municipios locales era de 1323 sin tener en cuenta los territorios no organizados, los pueblos nórdicos que dependen de la Administración regional de Kativik y las reservas autóctonas (Gouvernement du Quebec 2000: 13).

Los dos temas de mayor preocupación para la gestión pública local eran la extensión urbana y el desequilibrio fiscal que ello generaba. La definición que podemos ofrecer sobre la extensión urbana se centra en un doble proceso: por un lado, la expansión territorial de la zona urbanizada y, por otro, la desinversión de la zona de urbanización más antigua (generalmente localizada en el centro) o del centro histórico de la aglomeración. Las consecuencias de la extensión urbana son la dispersión de la población y de los lugares de actividad (trabajo, estudio, placer y servicios). Esto conlleva una mayor necesidad de planificación y de ordenación del territorio, pero de forma muy especial, requiere un enorme esfuerzo para seguir prestando unos servicios públicos locales cuya naturaleza y cantidad deben ser modificados para lograr ajustarse a esa disparidad económica y social que ha emergido de la extensión urbana. En Quebec esta problemática ha supuesto que entre 1980 y 1998 se hayan cerrado 47 escuelas y se hayan tenido que abrir 28 en los nuevos barrios.

Otra consecuencia que debemos señalar de la extensión urbana es el desequilibrio fiscal. El porcentaje de las tasas que debían pagar los habitantes de las ciudades centrales eran, a excepción de Baie-Comeau, siempre más elevados que los de los municipios de la periferia. Si estos datos los relacionamos con la renta media, podemos comprobar de nuevo, que las tasas por alojamiento eran mayores en la ciudad central que en la periferia. La renta 
media de los hogares de la ciudad de Quebec ascendía a 34.983 dólares en 1996, mientras que la media de los otros municipios de la región era de 47.415 dólares. Sin embargo, la ciudad de Quebec mantenía servicios que beneficiaban a toda la aglomeración en materia de policía, de equipamientos regionales y de alojamiento social.

El desequilibrio fiscal se agravó como consecuencia del papel que desempeñaba el impuesto sobre el territorio en el sistema fiscal local. Este impuesto incrementaba la concurrencia intermunicipal y hacía aún más difícil la emergencia de una dinámica de aglomeración. Esta situación empeoró en el momento en el que la base del impuesto sobre el territorio dejó de progresar (como había ocurrido en los años 80) para entrar en un período de retroceso en los años 90 .

En Quebec se había mantenido una política de subvenciones directas. Los municipios locales de menos de 1.000 habitantes recibían del Gobierno provincial una cantidad que ascendía a los 230 dólares por habitante. Esta cantidad suponía el 25\% del total de los ingresos de los presupuestos locales. Mientras que para los municipios de 5.000 habitantes o más, la cantidad percibida era de 35 dólares por habitante, es decir, el 3\% de los ingresos locales.

Lo sorprendente era que los municipios que se situaban en una franja de población que iba desde los más de 10.000 habitantes hasta los 100.000 no se beneficiaban, a penas, de las ayudas directas ni del Gobierno de Quebec ni de los Municipios Regionales de Condado y las Comunidades Urbanas. Mientras que a partir de los 100.000 habitantes los municipios obtenían unas ayudas muy elevadas, 196 dólares, como consecuencia de la ayuda gubernamental en materia de transporte en común y mantenimiento de las aguas. Esta medida constituía una de las principales razones por las que los representantes locales se oponían a la reagrupación municipal.

Si hacemos un breve recordatorio de las distintas políticas de reestructuración municipal llevadas a cabo por Quebec provincia se puede comprobar que estas generaron intensos debates pero ningún cambio profundo en la constitución de las áreas urbanas y rurales. Ya en los años 60 se impulsó una política de fusión de municipios de carácter voluntario que fue acompañada de incentivos fiscales. Esta política no tuvo ningún éxito, ya que no hubo una reducción significativa del número de municipios.

En los años 70, y con una táctica que abordaba caso por caso, se logró una reducción de número de municipios, de 1600 a 1500. En los 80, la prioridad fue establecida en el nivel regional con la creación de los Municipios Regionales de Condado (Municipalités Régionales de Comté) y la modificación de la división regional. Los Municipios Regionales de Condado fueron creados el 23 de septiembre de 1981 como consecuencia de la aprobación de la Ley 125 sobre la ordenación y el urbanismo de 6 de noviembre de 1979. Esta ley preveía que el espacio de Quebec habitado fuera enmarcado por 95 pequeñas regiones llamadas Municipios Regionales de Condado (86 en la actualidad). Estos territorios microregionales (supralocales) debían servir explícitamente de base para la emergencia de solidaridades y de iniciativas para el desarrollo social y económico. En 1987, se modificó la división de las regiones, tras intentar reforzar el proceso de descentralización iniciado en 1966 con la implantación de 10 regiones con un marcado carácter administrativo. El número actual de regiones es de 16 y su función principal es servir a la gestión de las actividades gubernamentales en todo el territorio (Proulx 1998: 118).

Por último, en 1995 se publicó el «Libro Verde» sobre la descentralización, Décentralisation: un choix de société. Con anterioridad, aunque no llegó a publicarse, se había elaborado el «Libro Blanco» sobre la descentralización en 1978. El «Libro Verde» encerraba la pretensión de dar un mayor protagonismo a otros niveles locales y regionales desde el Estado provincial, sobre todo, como consecuencia de un repliegue del Estado providencia (Collin et al. 1999: 48). Este nuevo impulso a la descentralización estaba estrechamente ligado al proyecto políti- 
co del Partido Quebequés que acababa de ganar las elecciones², la consecución de la independencia de Quebec. La descentralización como proceso quedó en letra muerta ya que el resultado del referéndum celebrado el 30 de octubre de 1995 dio la razón a la opción que defendía el statu quo de Quebec (49,6\% a favor de la independencia frente a un 50,4\% que votó por el no. La participación fue del $96 \%$ de los votantes inscritos). Los dos impulsos a la descentralización regional han estado promovidos por los gobiernos del Partido Quebequés y ligados a los resultados de los referendos que sometían a la población la cuestión de la separación de Quebec de la federación canadiense. En el anterior referéndum, el de 20 de mayo de 1980, los votos favorables al sí obtuvieron sólo el $40 \%$ y la tasa de participación fue del 85,6\%.

Todas las iniciativas referidas a la reestructuración municipal, refuerzo de las estructuras supramunicipales y proceso de descentralización de los distintos gobiernos provinciales han tenido repercusiones electorales. Quesnel propone, no como un factor determinante, que si las elites municipales entendían como demasiado radicales los cambios emprendidos por los gobiernos provinciales, éstos podían llegar a perder las elecciones. El Partido Liberal que estuvo en el poder desde 1960 hasta 1966 y desde 1970 hasta 1976, sufrió su derrota electoral como consecuencia de la creación de un nivel de gobierno en las regiones por encima de las principales áreas metropolitanas. La Unión Nacional que gobernó desde 1966 hasta 1970 decidió crear tres comunidades urbanas en las tres áreas metropolitanas principales y también perdió las elecciones en 1970. El Partido Quebequés tuvo como uno de los ejes centrales de sus gobiernos, desde 1976 hasta 1985 la reorganización municipal a través de procesos de descentralización y ello le condujo a su derrota electoral en 1985 (Quesnel 2000: 119).

En 1990 volvió al Gobierno provincial el Partido Liberal y reinició un proyecto de transferencia de responsabilidades y de costes a los municipios en dos aspectos tan fundamentales como el de la policía y las carreteras locales. El Partido Liberal fijó como objetivo principal de esta política las localidades pequeñas, sin embargo no logró el apoyo de sus alcaldes. Este Gobierno, ante el reto de las grandes urbes metropolitanas, no supo dar respuestas a las demandas de Montreal como área metropolitana a pesar de la realización del Informe Pichette en 1993. Tampoco hizo frente a las exigencias de las seis ciudades más importantes en materia de cooperación a una escala metropolitana.

En 1994, el Partido Quebequés toma el relevo en el Gobierno, y en 1996, desde el Ministerio de Asuntos Municipales, se impulsa un proyecto de fusión de municipios que se enmarca dentro de la política de consolidación de las instituciones locales.

En este contexto de reformas la región de Montreal merece una atención especial por su especificidad. La complejidad viene marcada por distintos factores: primero, territoriales, la expansión urbana a ambos lados del río San Lorenzo fragmenta el escenario; segundo, institucional, en un territorio en el que coexisten 100 municipios, 12 Regiones Metropolitanas de Censo y una Comunidad Urbana y, por último, económicos, dado el papel de locomotora de la economía y del desarrollo de la provincia que ejerce Montreal.

El Informe Pichette de 1993 fue uno de los intentos más serios de articular una respuesta a las demandas de Montreal. Este informe introdujo el concepto de «ciudad región» y pretendía reforzar el consenso regional para mejorar la cooperación intra-regional. Sus propuestas fueron rechazadas no sólo por los actores externos como la Asociación de Municipios Rurales y Regionales y los alcaldes de los municipios suburbanos, sino que algunos de los ministerios no vieron con buenos ojos la fusión de las cinco regiones administrativas del área de Montreal.

\footnotetext{
2 De forma muy resumida los resultados electorales de los dos principales partidos en los últimos 30 años en Quebec provincia los podemos presentar de la siguiente manera Partido Quebequés 1976-1985, 1994-1998, 1998-2003; Partido Liberal 1985-1989 y 1989-1994; 2003.
} 
La llegada del Partido Quebequés al poder tampoco empezó con muy buen pie en cuanto a la reforma del área metropolitana de Montreal. Al año (1997) tuvo que aceptar tras la oposición de los mismos protagonistas del rechazo del informe Pichette, que los Municipios Regionales de Condado no serían modificados y que la Comisión para el Desarrollo Metropolitano tendría carácter consultivo y no de decisión. Ante la celebración de elecciones anticipadas, el Gobierno prefirió posponer la creación de la Comisión para el Desarrollo Metropolitano, y el Partido Quebequés fue reelegido el 31 de noviembre de 1998. Entre otras medidas, el Gobierno decidió modificar el nombre y el contenido del Ministerio de Asuntos municipales para añadirle «y de la Metrópolis», dada la especificidad de Montreal.

La Comisión Nacional sobre las finanzas y el sistema fiscal local puesta en marcha en abril de 1998 antes de las elecciones provinciales publicó un informe en abril de 1999 conocido como el Informe Bédard. Este informe supuso un auténtico revulsivo y un cambio de las políticas llevadas a cabo hasta el momento para la reorganización municipal y supramunicipal, porque se basaba en 3 dimensiones fundamentales del gobierno municipal: en las estructuras (reforzar los 96 Municipios Regionales de Condado), las responsabilidades (estos organismos debían centrarse en la planificación y gozar de mayor autonomía en su financiación) y los procesos democráticos (consejos elegidos por sufragio universal directo).

Las reacciones no se hicieron esperar. La Asociación de Municipios Urbanos criticó que el informe Bédard se saliera del campo estricto de las finanzas y del sistema fiscal local. No le pareció bien que entrara en el debate sobre la reorganización estructural, rechazó el refuerzo de los Municipios Regionales de Condado y menos aún las posibilidades de elegir directamente a los gobiernos regionales.

Las reacciones de los alcaldes de Montreal y de Quebec fueron más suaves pero en la misma línea. La elección directa y el poder de tasación por parte de un segundo nivel de gobierno fueron las propuestas a las que más se opusieron.

La reacción del alcalde de Montreal, Pierre Bourque, consistió en volver a sacar a la luz el proyecto, de «una isla, una ciudad» (idea del alcalde Drapeau en los años sesenta). Este proyecto no obtuvo el apoyo de la Unión de municipios de las afueras (suburbanos) de la isla de Montreal, pero no fue descartada por la ministra Louise Harel.

Para la región de Quebec, el Informe Bédard proponía reconducir el número de municipios de 43 a 5. El alcalde de Quebec, Jean-Paul L'Allier, hizo una propuesta alternativa a finales de junio de 1999 que consistía en crear dos ciudades y una comunidad. Una ciudad para reagrupar el conjunto de municipios de la Comunidad Urbana de Quebec sobre la orilla norte del río San Lorenzo, mientras que la otra ciudad reagruparía la aglomeración urbana de la orilla sur. Estas dos ciudades se reencontrarían en el seno de una Comunidad urbana en la cual se añadirían los Municipios Regionales de Condado de la Jacques-Cartier y de la Côte-de-Beaupré.

En el verano de 1999, el diario de Montreal, Le Devoir, comenzó a publicar una serie de artículos en los que abogaba por la creación de una estructura para toda el área metropolitana de Montreal y la reorganización de los Municipios Regionales de Condado. Este terreno abonado por un aliado inesperado no fue desaprovechado por la Ministra de Asuntos Municipales y de la Metrópolis, Louise Harel, que arremetió con la necesidad de la reforma (Quesnel 2000: 124).

La ministra comenzó una serie de negociaciones con las dos principales asociaciones de municipios (la Asociación de Municipios Rurales y Regionales y la Asociación de Municipios Urbanos de Quebec), pero ante la imposibilidad de llegar a un acuerdo con los actores implicados en el proceso, el Ministerio decidió publicar el 
Libro Blanco (titulado La réorganisation municipale. Changer les façons de faire, pour mieux servir les citoyens), en marzo de 2000. Este documento contenía la política gubernamental para la reorganización municipal y supramunicipal.

Para cerrar este apartado podemos concluir que los intentos de reformas de la estructura municipal y supramunicipal tendentes a una mayor racionalización y reducción del número de municipios han estado envueltos en el fracaso. Las decisiones del cambio generalmente habían sido adoptadas de forma unilateral desde el Gobierno provincial, si bien, en distintas ocasiones estas decisiones venían avaladas por informes de Comisiones de expertos. Estos informes eran más radicales en sus propuestas de cambio que las propias iniciativas gubernamentales y, contrarias, a algunos de los actores implicados. El Informe Bédard jugó un importante papel no tanto por sus propuestas sino por las reacciones que surgieron en torno a ellas. En primer lugar, porque del rechazo que suscitó se dibujaron los actores que iban a estar en contra (como era el caso de las Asociaciones de Municipios Rurales y Regionales, la Asociación de Municipios Urbanos y los alcaldes de los municipios suburbanos), y a favor de la política gubernamental del Partido Quebequés (la prensa escrita y los alcaldes de las grandes ciudades). En segundo lugar, surgieron dos propuestas alternativas al Informe Bédard por parte de los alcaldes de Montreal y Quebec que sirvieron de base para la reorganización municipal y supramunicipal. Por último, hemos intentado poner de manifiesto la relación entre las reformas locales cuestionadas por las elites locales y el fracaso del partido en el poder en las convocatorias electorales.

\section{Las fusiones y el refuerzo de las estructuras supramunicipales. El «Libro Blanco»}

El «Libro Blanco» eligió dos objetivos en cuanto al foco de la reorganización: por un lado, la reagrupación o fusión de los municipios locales y por otro, reforzar los Municipios Regionales de Condado y las aglomeraciones urbanas, en especial, poner en marcha las comunidades metropolitanas en las aglomeraciones de Montreal, Quebec y Outaouais. Las reagrupaciones se producirán con carácter preferente en las aglomeraciones de Quebec, Montreal y Outaouais.

La finalidad de esta política consiste en encontrar un nivel administrativo apto para solucionar los problemas de la gestión pública local. Las Comunidades Urbanas (nivel supramunicipal) habían desempeñado un papel muy importante, en especial, en aquellos aspectos regionales de gestión centrados en la depuración de las aguas y el transporte en común. Sin embargo, eran unas estructuras que no respondían a las necesidades actuales y, sobre todo, que su territorio se había quedado demasiado reducido, ya que sólo contaba con parte de las aglomeraciones urbanas. Lo mismo ocurría con los Municipios Regionales de Condado que habían servido para la planificación de la ordenación del territorio y como lugar para la puesta en común de los servicios, sin embargo, no habían generado la emergencia de un «espíritu regional». Entre otras razones porque los municipios disponían de la capacidad de retirarse de estas estructuras lo que las dotaba de un carácter temporal. No existían unas instancias de decisión fuertes que coincidieran con las grandes aglomeraciones.

La política de reagrupación es una tendencia que se puede encontrar en otras provincias canadienses para sus grandes ciudades como Toronto y Ottawa, en Estados Unidos, y en Francia, cuya última propuesta de cooperación supramunicipal es la creación de las Comunidades de Aglomeración. En los otros casos canadienses, las fusiones también habían sido fruto de una decisión gubernamental provincial incluso contra la opinión de la población y de los consejos municipales (Ontario).

La estrategia prevista por el Ministerio era primero negociar el plan de actuación con las tres comunidades urbanas para después extender el acuerdo a los Municipios Regionales de Condado y al resto de municipios. 
El calendario de aplicación era bastante apresurado con los siguientes pasos marcados: en abril la publicación del «Libro Blanco», en junio la publicación de la legislación necesaria para su puesta en marcha, la implementación del plan a partir de enero de 2001 y en enero de 2002 la abolición de la tasa de redistribución (las subvenciones dadas a los pequeños municipios).

Las primeras reacciones al «Libro Blanco» vinieron de las asociaciones de Municipios Rurales y Urbanos así como de los alcaldes de los municipios suburbanos. Para el Presidente de la Asociación de Municipios Urbanos de Quebec, el plan de fusiones implicaba dos graves problemas. Por un lado incrementaría los costes con la inmediata traducción de un aumento de las tasas y, por otro, provocaría un problema en materia laboral, al tener cada municipio condiciones laborales y convenios colectivos distintos.

A pesar de estas manifestaciones en contra, el Parlamento creó la Comisión Metropolitana del Gran Montreal. En noviembre de 2000 el Gobierno presenta un proyecto de ley (170) para la fusión de municipios en las cinco áreas metropolitanas mayores de la provincia para dar paso a la creación de cinco grandes ciudades:

- Montreal, con 1,8 millones de habitantes y 28 municipios fusionados.

- Quebec, con 504.000 habitantes y 13 municipios fusionados.

- Longueuil, 380.000 habitantes y 8 municipios fusionados.

- Gatienau-Hull, 200.000 habitantes y 5 municipios fusionados.

- Lévis, 118.000 habitantes y 10 municipios fusionados.

Los únicos que apoyaron la política del Gobierno provincial fueron los principales periódicos, salvo La Gazette, que estaba en contra de las fusiones obligatorias porque se sentía más próxima de las posturas de la población anglófona.

Especialmente en Montreal, la fusión implicaba una dimensión añadida, la lingüística, ya que 14 de los 28 municipios llamados a fusionarse en la isla de Montreal gozaban de un estatuto bilingüe tal y como lo establecía la Carta de la Lengua Francesa de 1977. En Quebec existían 91 pueblos con este estatus especial por contar con una mayoría de ciudadanos que hablaban otra lengua distinta a la francesa (denominados «alófonos»). Los anglófonos sentían que la fusión lesionaba sus derechos (utilizar su lengua en su denominación oficial así como en las comunicaciones internas; utilización de su lengua en carteles públicos y en el lugar de trabajo), y que se diluía su presencia en una comunidad con una masa crítica francófona más numerosa y fuerte (Sancton 2000: 45).

Salvo el papel jugado por los diarios, los otros actores que estaban a favor de las fusiones no desempeñaron un papel activo. Las ciudades centrales, algunos grupos de feministas y ciertos grupos de ciudadanos socialdemócratas llevaron a cabo algunas manifestaciones a favor de la política gubernamental.

Los dos objetivos de la política de reorganización municipal del gobierno de Quebec, los reagrupamientos urbanos y el refuerzo de las estructuras supramunicipales como los Municipios Regionales de Condado y las Comunidades Metropolitanas, no han supuesto una reforma tan radical como se temía en un principio:

- De 1.328 municipios se ha pasado a 1.110 (en enero de 2006 ascenderán a 1141 como consecuencia de la reconstitución).

- Los Municipios Regionales de Condado permanecen prácticamente inalterados en sus funciones (con la previsión de tener unas competencias supeditadas a las Comunidades Metropolitanas si su territorio está dentro de ellas), sólo su territorio se ha modificado por algunas fusiones.

- La verdadera transformación se ha concentrado en las Comunidades Metropolitanas que al final se han creado sólo en Montreal y Quebec. 
En ninguna de las estructuras supramunicipales se refuerza sus consejos a través de la elección por sufragio universal directo. Tras la última reforma de la política de reconstitución de municipios en los Municipios Regionales de Condado (los que no pertenezcan a la Comunidad Metropolitana de Montreal), pueden elegir a su prefecto a través de sufragio universal directo. Hasta la fecha sólo siete lo han hecho así. Por último, podemos señalar que los antiguos municipios fusionados han sido sustituidos por distritos. Es decir, que persiste la necesidad de una gestión de proximidad, a pesar de los intentos de ahorro y de racionalización con la reducción del número de representantes locales.

\section{La Comunidad Metropolitana de Quebec}

Según la ley 170 las Comunidades Metropolitanas deben ser creadas en aquellas aglomeraciones en las que ya existe una comunidad urbana. El territorio de las Comunidades Metropolitanas, que debe coincidir con el de las Regiones Metropolitanas de Censo, no afectará a la división actual de los Municipios Regionales de Condado. Es más, se dará la situación en la que parte del territorio de un Municipio Regional de Condado esté dentro de la Región Metropolitana de Censo y otra parte fuera.

Los consejos de las comunidades metropolitanas no serán elegidos por sufragio directo, y por esta razón no tendrán acceso directo a la tasación sobre el territorio. Por tanto, su financiación se nutre gracias a las cuotas partes que aportan los municipios. Otras fuentes de financiación serán, además de las ayudas gubernamentales, las rentas obtenidas de un programa de reparto del crecimiento del porcentaje de la base imponible del impuesto sobre el territorio, los derechos de matriculación, la tasa sobre la gasolina (percibida por el Gobierno pero devuelta a las Comunidades Metropolitanas para sufragar el transporte en común) y el pago de peajes o estacionamientos.

A pesar de las declaraciones de intenciones sólo se han creado dos Comunidades Metropolitanas, la de Montreal en enero de 2001 y la de Quebec en enero de 2002. Su consejo está compuesto por los representantes municipales de los municipios locales que la constituyen.

El Consejo de la Comunidad Metropolitana de Quebec lo conforman 17 miembros (el alcalde de Quebec, el alcalde de Lévis, 8 consejeros de Quebec, 4 consejeros de Lévis, el prefecto del Municipio Regional de Condado de la Côte-de-Beaupré, el prefecto del Municipio Regional de Condado de la Île-d'Orléans y el prefecto del Municipio Regional de Condado de la Jacques-Cartier), y al igual que en Montreal, el alcalde de la Ciudad de Quebec es el que preside el Consejo de la Comunidad Metropolitana.

La Comunidad Metropolitana de Quebec es un organismo de planificación y concertación que pretende una mayor cohesión entre la Ciudad de Quebec, la Ciudad de Lévis y los municipios que forman parte de los Municipios Regionales de Condado de la Jacques-Cartier, de la Côte-de-Beaupré y de la Île-d-Orléans. En total la Comunidad Metropolitana reagrupa a 26 municipios. Su estructura como institución presenta un carácter ligero centrada especialmente en la planificación y no en la ejecución.

A los 3.350 kilómetros cuadrados del territorio sobre los 26 municipios hay que sumarles los Territorios No organizados, hasta alcanzar la cifra de 9.500 kilómetros cuadrados. La población asciende a las 702.000 personas, es decir, el 10\% de la población total de Quebec provincia (Ministère des Affaires Munipales des Régions 2005).

Sobre el territorio de la Comunidad Metropolitana coexisten dos regiones administrativas, la región de la Capital-Nacional sobre la orilla norte del río San Lorenzo y la región de la Acudière-Appalaches sobre la orilla sur. 
El Consejo de la Comunidad compuesto por 17 miembros está apoyado en su trabajo por un comité ejecutivo, por cuatro comisiones consultivas permanentes (ordenación y gestión del territorio, transporte en común metropolitano, desarrollo económico y turístico, y medio ambiente) y por un consejo consultivo agrícola. La estructura administrativa cuenta tan sólo con 16 empleados en 2003, para mantener el compromiso de una institución ligera y su presupuesto de funcionamiento asciende a un total de 2,8 millones de dólares (Communauté Métropolitaine de Quebec 2004: 6).

Según Carrier y Gingras (2003: 34 y 35) se pueden reagrupar los mandatos establecidos por ley a la Comunidad Metropolitana de Quebec en 5 campos de competencia: ordenación del territorio y transporte en común metropolitano; desarrollo económico, turístico e internacional; desarrollo social; desarrollo del medio ambiente y, desarrollo cultural. Los poderes conferidos a la Comunidad pueden ser resumidos en cuatro funciones principales: planificación, armonización, promoción y financiación.

Si cruzamos estas dos categorías podemos obtener las funciones que le han sido asignadas según los campos de acción o competencias de la Comunidad Metropolitana de Quebec.

\section{TABLA 1}

Relación entre las Competencias y las Funciones asignadas a la Comunidad Metropolitana de Quebec

\begin{tabular}{|l|c|c|c|c|}
\hline \multicolumn{1}{|c|}{ Campos de competencia } & \multicolumn{2}{c|}{ Funciones de la Comunidad Metropolitana de Quebec } \\
\cline { 2 - 5 } & Planificar & Armonizar & Promover & Financiar \\
\hline 1. Ordenación del territorio y transporte en común metropolitano & $\mathrm{X}$ & $\mathrm{X}$ & & $\mathrm{X}$ \\
\hline 2. Desarrollo económico, turístico e internacional & $\mathrm{X}$ & $\mathrm{X}$ & $\mathrm{X}$ \\
\hline 3. Desarrollo social & $\mathrm{X}$ & & & \\
\hline 4. Desarrollo del Medio Ambiente & $\mathrm{X}$ & & & \\
\hline 5. Desarrollo cultural & $\mathrm{X}$ & & & $\mathrm{X}$ \\
\hline
\end{tabular}

Fuente: Carrier y Gingres (2003: 34-35).

El Informe de los años 2003-2004 presentado por la Comunidad Metropolitana de Quebec resalta como uno de las primeras realizaciones concretas, la implantación del proyecto piloto «laissez-passen» (paso libre) metropolitano con el fin de permitir a los usuarios de las redes de transporte en común de la orilla sur y de la orilla norte desplazarse con un solo pase. (Communauté Métropolitaine de Quebec 2004: 11). Los esfuerzos durante esos dos primeros años de andadura han estado destinados a la comunicación con la finalidad de fomentar un espíritu metropolitano, demostrar las capacidades de esta institución y, en especial, la creación de una identidad visual.

El territorio de intervención de la Comunidad Metropolitana de Quebec se encuentra en una fase de integración espacial progresiva (Barbonne et al. 2003: 6). No podemos olvidar que el territorio urbanizado de la región metropolitana de Quebec se ha extendido a lo largo de los años. Las consecuencias principales han sido una mayor desconcentración espacial de la población, así como de los empleos. La tendencia es un descenso demográfico de los municipios del este de la aglomeración (el Municipio Regional de Condado de Côte-de- 
Beaupré) y un desplazamiento del centro de gravedad demográfico hacia el oeste. El crecimiento del empleo es más rápido que el de la población, y el empleo se desconcentra igualmente más rápido. Aún así, el empleo permanece más concentrado que la población en 2001. Sin embargo, los polos comerciales permanecen localizados principalmente en la orilla norte. Los polos de educación están fuertemente reagrupados en el centro de la aglomeración. Por último, las actividades ligadas al entretenimiento permanecen muy dispersas dado la diversidad de actores y de equipamientos implicados. Todos estos factores hacen más difícil la función de planificación y ordenación de la Comunidad Metropolitana.

En cuanto a la Ciudad de Quebec como entidad distinta a la Comunidad Metropolitana querríamos dedicarle algunas líneas para explicar su funcionamiento y sus principales características.

El Consejo de la ciudad de Quebec está compuesto por el alcalde y 39 consejeros. El alcalde es elegido por los electores de todos los distritos, mientras que los consejeros son elegidos por los electores del distrito electoral que representan (Chabot Grant Thorton 2004: 5). La elección del alcalde y de los consejeros se hace a través de sufragio universal directo, mediante unas elecciones que se celebran cada 4 años de forma simultánea a partir de 2005. La tasa media de participación es del 54\%. Hay algunas cuestiones que pueden ser sometidas a referéndum por los municipios como el urbanismo o la petición de un préstamo por la corporación local.

La ciudad contará también con consejos de distrito y de barrio. En la actualidad, han sido creados 11 consejos de barrio. Además del Consejo de las Artes de Quebec, la Ciudad ha puesto en marcha numerosos comités compuestos por representantes, funcionarios de la Ciudad y ciudadanos como el comité responsable de la toponimia, o de desarrollo económico, de las mujeres y la ciudad, etc.

Las competencias de la Ciudad de Quebec se centran en los siguientes campos: ordenación del territorio y urbanismo; desarrollo comunitario, económico, cultural y social; la eliminación de materias residuales; la cultura; los entretenimientos y los parques; alojamiento social; redes arteriales; limpieza de la atmósfera y las aguas; suministro de agua potable; promoción y acogida turística y creación del tribunal municipal.

La Ciudad de Quebec ha realizado esfuerzos por mejorar su gestión y orientarla hacia la mejora de sus rendimientos. Así, en la primavera de 2003 la Ciudad ha propuesto un proyecto de reorganización administrativa en el que se prevé la realización de un contrato de la Ciudad de Quebec con el Gobierno. El objetivo de este contrato es lograr un ahorro presupuestario de 8 millones de dólares por año durante los próximos cinco años (2004-2008).

La gobernabilidad metropolitana en el caso de la Comunidad Metropolitana de Quebec debe hacer frente a una serie de desafíos que pueden ser divididos en dos categorías como presentamos en la Tabla 2, p. 148.

En estas líneas hemos intentado poner de manifiesto de forma muy resumida que el papel que se le ha dado a las Comunidades Metropolitanas está destinado más a la planificación y a la concertación que a la ejecución. Una de las razones ha sido para evitar la creación de una estructura administrativa que hiciera sombra a la ciudad que acababa de ser reagrupada. Las grandes ciudades son las que controlan en definitiva los consejos de las Comunidades Metropolitanas y también de las Aglomeraciones como veremos en el siguiente epígrafe. Por tanto, la crítica realizada a las estructuras supramunicipales como entes alejados de los ciudadanos, poco transparentes, y con un grave déficit democrático no ha sido solventada con la actual reforma. Las Comunidades Metropolitanas desde la perspectiva de la gobernabilidad se enfrentan a un importante reto que es el de establecer los mecanismos necesarios para la negociación y la concertación entre una serie de actores de distinta natu- 
TABLA 2

Desafíos a la gobernabilidad de la Comunidad Metropolitana de Quebec

\begin{tabular}{|c|c|c|}
\hline \multicolumn{1}{|c|}{ Desafíos territoriales } & Desafíos operacionales \\
\hline $\begin{array}{c}\text { Cohabitación del medio rural con el medio urbano (una fuerte proporción } \\
\text { del territorio de la Comunidad Me-tropolitana de Quebec está ocupada } \\
\text { por espacios rurales). }\end{array}$ & Reparto de poder y relaciones basadas en consenso es difícil. \\
\hline $\begin{array}{c}\text { La división por el Río San Lorenzo entre la orilla Norte (Quebec ciudad) y } \\
\text { la orilla sur (Lévis). Las relaciones entre las dos ciudades habían estado } \\
\text { marcadas por la competencia. }\end{array}$ & $\begin{array}{c}\text { La unidad es difícil de mantener entre los distintos actores, especialmente } \\
\text { entre las dos orillas. Necesidad de implantar procesos permanentes de } \\
\text { discusión. }\end{array}$ \\
\hline $\begin{array}{c}\text { Fragmentación municipal previa generaba tensiones. La integración aún es } \\
\text { muy reciente y no ha hecho desaparecer todas las resistencias. }\end{array}$ & $\begin{array}{c}\text { Conflictos de valores dada la pluralidad de actores. Los valores son difícil- } \\
\text { mente negociables. }\end{array}$ \\
\hline $\begin{array}{c}\text { El entorno regional de la Comunidad Metropolitana de Quebec (fomentar } \\
\text { las relaciones, la comunicación y la cooperación con regiones contiguas). }\end{array}$ & $\begin{array}{c}\text { La democracia. La aparición de otros actores en la escena pública cuestiona } \\
\text { la legitimidad democrática de la gobernabilidad. De ahí, la necesidad de } \\
\text { incorporar una aproximación inclusiva (especialmente a los ciudadanos). }\end{array}$ \\
\hline
\end{tabular}

Fuente: Carrier y Gingras (2003: 11).

raleza (municipios reagrupados en grandes ciudades, municipios regionales de condado, municipios reconstituidos, representantes locales con inercias localistas y actores de la sociedad civil), pero sobre todo con intereses muy diferentes y unas demandas cada vez más heterogéneas.

\section{Las políticas de reconstitución}

La finalidad de este apartado consiste en analizar el reciente proceso de reconstitución iniciado como consecuencia de la llegada al poder del Partido Liberal el 14 de abril de 2003.

El 6 de junio de 2003, los liberales de Jean Charest, recién llegados al Gobierno de Quebec, presentan a la Asamblea Nacional el proyecto de ley 1. ${ }^{\circ}$, que autoriza a los ciudadanos librarse de las fusiones municipales decretadas por el anterior gobierno formado por el Partido Quebequés y liderado por Lucian Bouchard. Esta medida es la traducción de una de las promesas electorales que llevó al Partido Liberal al poder.

Esta ley permite a los ciudadanos contrarios a las fusiones recurrir a una serie de mecanismos políticos. Principalmente, el mecanismo consiste en la celebración de referéndum (con unos requisitos muy exigentes), regulado por la ley 9, de 17 de diciembre de 2003, que se celebraría el 20 de junio de 2004.

En la política de reorganización municipal se pueden señalar en esta década dos grandes fases:

- 2000-2003: Reagrupaciones:

- 213 municipios y partes de municipios son reagrupados para formar 42 nuevos municipios.

- El número de municipios en Quebec pasa de 1328 a 1110.

- Se implantan dos Comunidades Metropolitanas.

- 2003-2005: Reconstitución:

- 31 de los 213 municipios reagrupados han elegido reencontrar su territorio.

- El número de municipios pasa, por tanto, de 1110 a 1141 el 1 de enero de 2006. 
- Esto conlleva la puesta en marcha de 11 Consejos de Aglomeración (para mantener las relaciones entre el nuevo municipio que se desfusiona y la aglomeración de la cual se desgaja).

La reconstitución de antiguos municipios ha obligado a generar una nueva figura, la aglomeración. Las aglomeraciones creadas son: Cookshire-Eaton, La Tuque, Les Iles-de-la-Madeleine, Longueuil, Mont-Laurier, Montreal, Mont-Tremblant, Québec ${ }^{3}$, Rivière-Rouge, Sainte-Agathe-des-Monts y Sainte-Marguerite-Estérel (Ministère des Affaires Municipales et des Régions 2005).

Las competencias de los Consejos de Aglomeración son: los elementos de la seguridad pública (servicios de policía, seguridad civil y seguridad contra incendios); la evaluación municipal; el transporte colectivo de personas; las vías de circulación que constituyen la red arterial en el territorio de la aglomeración; la alimentación y el tratamiento de las aguas para las aglomeraciones de más de 100.000 habitantes; y la eliminación y reciclaje de materias residuales así como la elaboración y adopción de un plan de gestión de estas materias.

Los antiguos municipios reconstituidos deben asumir los gastos relativos a las competencias locales para su gestión, los gastos de financiación de las competencias de la aglomeración y las actividades compartidas con el municipio central. Pero también queda claro, que los costes de las transiciones son asumidos por los nuevos municipios reconstituidos.

En este sentido, hay un aspecto que no deja de llamarnos la atención, pues, una vez que un municipio logra romper sus vínculos de reagrupamiento con los otros no puede volver a sus estatus anterior, es decir, que no recupera sus poderes originales. Sólo puede tener un consejo con competencias que afectan a cuestiones de proximidad (calzada local, alcantarillado, actividades lúdicas y emisión de permisos). Además, debe formar parte de un Consejo de Aglomeración que sirve de nexo entre la ciudad fusionada y los municipios reconstituidos. La capacidad de decisión dentro del Consejo está inclinada favorablemente hacia la gran ciudad y no sobre los municipios reconstituidos.

La atribución de votos se hace en función del peso demográfico de cada municipio miembro. Por tanto, la ciudad fruto de la reagrupación de municipios es la que tiene más votos y detenta el control sobre las decisiones políticas. En el Consejo de Aglomeración de Montreal compuesto por los 14 municipios reconstituidos y la ciudad de Montreal, el alcalde de esta última ciudad dispone del 87\% de los votos. Mientras que para Quebec, la ciudad de Quebec dispone del 96\% de los votos, frente a los dos municipios reconstituidos, l'Ancienne Lorette y Saint-Augustin-de-Desmaures (Gravel y Huard 2006: 23). No obstante, la ley 75 de 2004 que regula el ejercicio de ciertas competencias en ciertas aglomeraciones, permite a los municipios reconstituidos oponerse a una decisión adoptada por el Consejo de Aglomeración. Con esta medida, se introduce no sólo un freno a la agilidad de la gestión pública local, pues ya hay diversos casos de bloqueo del presupuesto local (el más grave es el de Longueuil), sino que además introduce al Ministerio de Asuntos Municipales y de la Región como árbitro dentro de la esfera local.

En las grandes ciudades fusionadas (Montreal, Quebec, Longueuil, Lévis, Saguenay y Sherbrooke), se han instaurado los distritos como un mecanismo para mantener la gestión de proximidad. Los consejeros de estos distritos son elegidos por sufragio universal directo. En Montreal, a partir de 2003, gracias al impulso de su alcalde Tremblay bajo la presión de su partido (entre sus miembros se encuentran alcaldes de municipios suburbanos caracterizados por su oposición frontal a la política de reagrupación municipal), ha reforzado de forma muy espe-

${ }^{3}$ Los dos municipios reconstituidos de Quebec son L'Ancienne Lorette y Saint-Augustin-de-Desmaures. 
cial a los distritos, concediéndoles unas competencias similares a las de los municipios (en materia de ordenación y urbanismo, y gestión de recursos humanos y financieros) (Gravel y Huard 2006: 23). Para algunos autores como Serge Belley, la instauración de los distritos habría sido una medida adoptada por el gobierno Charest para frenar su propia política de reconstitución, dada la complejidad que ésta implicaba. La pretensión era conceder amplios poderes a estos distritos para persuadirlos de la falta de necesidad de implicarse en el proceso de reconstitución, dados los costes, el poco tiempo transcurrido desde la fusión y el miedo a generar una estructura más, el consejo de aglomeración. Esta estrategia no ha tenido mucho éxito pues 14 de los 28 municipios fusionados en Montreal han conseguido reconstituirse.

En cualquier caso, la reconstitución ha sido un proceso costoso, complicado porque genera incluso otra instancia de decisión y de ejecución más con la instauración de la aglomeración y, además, los municipios reconstituidos no recuperan su estatus anterior. Probablemente todos estos factores son los que han hecho enfriar las expectativas de reconstitución de los antiguos municipios y percibir de una forma más positiva las fusiones. Ante la posibilidad de romper con las fusiones forzosas no ha habido una reacción masiva, sólo en Montreal, 14 de los 28 municipios reagrupados han conseguido separarse, todos aquellos municipios que tenían el estatuto de bilingües.

Todo esto pone de manifiesto que el giro dado por el Partido Liberal a la estructura político-administrativa local y supralocal no respondía a criterios de eficacia y racionalidad en la gestión pública, sino a criterios de carácter político, principalmente, cumplir con la promesa electoral dada a su electorado (conformado por un importante componente de anglófonos), de acabar con las fusiones.

\section{Conclusiones}

Tras este recorrido podemos avanzar algunas conclusiones en función de los tres ejes que vertebraban el objeto de análisis de este artículo, la política de reorganización municipal y supramunicipal. Si comenzamos por el segundo eje que se preocupaba por el grado de descentralización de esta política, podemos afirmar que las Comunidades Metropolitanas son unos entes administrativos con un grado de descentralización muy débil. Las competencias que le han sido asignadas se centran más en aspectos de planificación y promoción que en la ejecución. No disponen de un sistema fiscal propio ni de una capacidad impositiva y su órgano de decisión es elegido por sufragio indirecto. Las aglomeraciones no han hecho más que generar una mayor complejidad y reforzar las críticas que señalan la opacidad y el alejamiento de los centros de toma de decisiones de los ciudadanos.

La instauración de los distritos en algunas de las ciudades fusionadas pone de manifiesto dos realidades. La primera es la necesidad de una gestión de proximidad, en donde el ciudadano controle y conozca a sus representantes y, la segunda es que esta creación supone un intento del Gobierno del Partido Liberal de frenar su propia política de reconstitución. La otra vía que se ha reformado para acallar las críticas ante la organización de las estructuras supramunicipales ha sido el modo de elección del prefecto de los Municipios Regionales de Condado. Como ya hemos indicado, éste puede ser elegido por sufragio universal directo (excepto en los 10 Municipios Regionales de Condado de Montreal), pero es una vía novedosa que hace sospechar que los Municipios Regionales de Condado probablemente van a estar llamados a desempeñar un papel más importante en un posible refuerzo del proceso de descentralización.

En cuanto a los ejes primero (las motivaciones formales y declaradas de la política de reorganización municipal permanecen en el campo de la gestión y no de la política) y tercero (la legitimidad de la Administración se 
refuerza en la actualidad principalmente, por los rendimientos), podemos afirmar que están estrechamente vinculados. Los criterios como la eficacia, la eficiencia y la calidad han estado presentes en la política de reorganización municipal y supramunicipal y se inscriben dentro de la búsqueda de la legitimidad de la Administración por los rendimientos. Sin embargo, estos no son los únicos objetivos presentes en estas reformas, ni los que determinan los cambios. El factor político, envuelto por la cuestión de la lengua, aparece constantemente como un vector explicativo de los cambios en la esfera local. No queda más remedio que admitir que la necesidad de la legitimidad institucional de la Administración y su estrecha relación con la esfera política son inevitables para comprender la política de reorganización municipal y supramunicipal y la de reconstitución.

\section{REFERENCIAS BIBLIOGRAFICAS Y DOCUMENTALES}

Gouvernement de Quebec, (2000), La réorganisation municipale. Changer les facons de faire, pour mieux servir les citovens. Quebec: GQ.

Comité de Transition de la Ville de Quebec, (2002), Quebec, ville nouvelle: compte rendu des travaux du Comité de transition. Quebec: CTVQ.

Barbonne, R. et al. (2003), Le territoire d'intervention de la Communauté Métropolitaine de Quebec. Ses limites et son intégration. Quebec: Université Laval, en: http://www.cmquebec.qc.ca/pub.php?PubCat=4 (Consulta: 6 de abril 2006).

Benoit, V. y Méllevec, A. (2003), «Les fusions municipales au Quebec», Powvirrs Locanx, 57: 116-122.

Bourret, A. (2001), La place de la décentralisation dans le regroupement municipal an Quebec en 2001, Thèse de maitrise. Quebec: Université Laval.

Carrier, M. y Gingras, P. (2003), La gowvernance métropolitaine à la Communauté Métropolitaine de Quebec: principes et procédures. Quebec: Université Laval. http://www.cmquebec.qc.ca/pub.php?PubCat=4 (Consulta: 6 de abril 2006).

Chabot Grant Thorton, R. (2004), Étude sur les consequences et les coûts de la reconstitution eventuelle des anciennes municipalities de l'actuelle ville de Quebec. Quebec: Ville de Quebec, en: http://www.quebec-transition.qc.ca/IMG/pdf/queb_etud.pdf (Consulta: 21 de noviembre de 2005).

Clark, J. y Newman, J. (1997), The Managerial State: Power, Politics and Ideology of Social Welfare. London: Sage.

Collin, J P. et al. (2000), «Rive-Sud de Montreal, dynamique intermunicipale et integration metropolitaine», Urban History Review. 18,. 2: 53-60.

Commisssion Nationale sur les Finances et la Fiscalité Locale (1999), Pacte 2000: rapport abrégée. Quebec: Ministère des Affaires Municipales.

Communaté Métropolitaine de Quebec (2004), Rapport annuel.

Desbiens, J. dir., (1996), La taille idéale pour offrir les services municipaux an Quebec: pour tout savoir sur les avantages et les inconvénients économiques des fusions de municipalités et des regroupements de services. Chicoutimi: Groupe Jacques Desbiens.

Desbiens, J. (1999), Fusions municipalités et économies d'échelle: mythes et réalités. Chicoutimi: Groupe Jacques Desbiens.

Divay, G. (2002), Le monde municipal quebecois 2000-2012; changer les façons de voir pour changer les façons de faire: élements condensés pour une prospective. Montréal: Institut National de la Recherche Scientifique. Urbanisation, culture et société.

Gagné, S. (2000), «Une solution qui passe ou qui casse pour Montréal?: projet une île, une ville», La Revue municipale et des travaux publics, $78,4: 19-20$.

Gelinas, A. (2002), L'intervention et le retrait de l'État: l'impact sur l'organisation gouvernamentale. Quebec: Presses de L'Université Laval.

Laforest, G. Montigny, E. (2005), «Le fédéralisme exécutif, problèmes et actualités», en Pelletier, R. y Tremblay, M. (2005), Le parlementarisme canadien, Quebec: Presses de l'Université Laval. 
Mania, M. (2000), The Assault on Local Government. Montreal y Kingston: McGill-Queen's University Press.

Ministère des Affaires Municipales des Régions (2005), L'Organisation municipale du Quebec, en: http://www.mamr. gouv.qc.ca/publications/organisation/org_mun_qc_fra.pdf. (Consulta: 7 de noviembre de 2005).

Proulx, M.-U. (1998), «L'organisation des territoires au Quebec: le débat sur la décentralisation gouvernementale». L'Action nationale, vol. 88. 2: 97-160.

Quesnel, L. (2000), «Municipal Reorganisation in Quebec», Canadian Journal of Regional Science, XXIII: 1. Spring: 115-134.

Sancton, A. (1999), «Differing approaches to municipal restructuring in Montreal and Toronto: from the Pichette Report to the Greater Toronto Services Board», Canadian Journal of Regional Science, vol 22. 1-2: 187-199.

- (2000), La frénesie des fusions : une attaque à la démocratie locale? Montréal: McGill-Queen's University Press.

Tindal, C. R. y Nobes Tindal, S. (1995), Local government in Canada. Toronto: McGraw-Hill Ryerson.

Université Laval, (2002), Quebec, ville nowvelle: compte rendu des travaux du Comité de transition. Quebec: Centre d'Analyse des Politiques Publiques. 


\section{Documentos}

